\title{
On the Mathematics of Mathematical Handwriting Recognition
}

\author{
Stephen M. Watt \\ University of Western Ontario \\ London, Canada \\ www.csd.uwo.ca/ $\sim_{\text {watt }}$
}

\begin{abstract}
Accurate computer recognition of handwritten mathematics offers to provide a natural interface for mathematical computing, document creation and collaboration. Mathematical handwriting, however, provides a number of challenges beyond what is required for the recognition of handwritten natural languages. For example, it is usual to use symbols from a range of different alphabets and there are many similar-looking symbols. Many writers are unfamiliar with the symbols they must use and therefore write them incorrectly. Mathematical notation is two-dimensional and size and placement information is important. Additionally, there is no fixed vocabulary of mathematical "words" that can be used to disambiguate symbol sequences. On the other hand, there are some simplifications. For example, symbols do tend to be well-segmented. With these charactersitics, new methods of character recognition are important for accurate handwritten mathematics input.
\end{abstract}

We present a geometric theory that we have found useful for recognizing mathematical symbols [1], [2], [3]. Characters are represented as parametric curves approximated by certain truncated orthogonal series. This maps symbols to the lowdimensional vector space of series coefficients. The Euclidean distance in this space is closely related to the variational integral between two curves and may be used to find similar symbols very efficiently. Training data sets with hundreds of classes are seen to be almost linearly separable, allowing classification by ensembles of linear SVMs [4], [5]. In this setting, we find it particularly effective to classify symbols by their distances under various norms to the convex hulls of nearest neighbors from known classes [3]. By choosing the functional basis appropriately, the series coefficients can be computed in real-time, as the symbol is being written [6], [7]. Using truncated series for integral invariant functions, orientation- and shear-independent recognition is achieved [8], [9]. We have seen that the distances to the SVM separating planes or to the convex hulls of nearest neighbors provide a reliable confidence measure for classifications [10]. This allows the combination geometric recognizers with $n$-gram based recognizers. To this end we can use statistical information from corpora of mathematical research papers and university engineering mathematics texts [11], [12], [13]. The relative frequency of symbols depends on the mathematical domain, and can even be used to find subject classification of mathematical documents [14]. We are currently investigating how orthogonal series representations may be used to compress ink traces in a form that may allow recognition without decompression of the database. Preliminary work on this problem is reported in [15].

We find this geometric appraoch, based on distances in a space of functional approximations, quite appealing. It gives a single, coherent view and several related techniques with remarkably high recognition rates that do not rely on peculiarities of the symbol set.

\section{REFERENCES}

[1] B.W. Char and S. M. Watt, "Representing and Characterizing Handwritten Mathematical Symbols Through Succinct Functional Approximation", pp. 1198-1202, Proc. International Conf. on Document Analysis and Recognition, (ICDAR 2007), IEEE Press.

[2] O. Golubitsky and S. M. Watt, "Online Recognition of Multi-Stroke Symbols with Orthogonal Series", pp. 1265-1269, Proc. 10th International Conf. on Document Analysis and Recognition, (ICDAR 2009), IEEE Computer Society.

[3] O. Golubitsky and S. M. Watt, "Distance-Based Classification of Handwritten Symbols", International Journal of Document Analysis and Recognition, 13 (2) pp. 133-146, June 2010, Springer.

[4] B. Keshari and S. M. Watt, "Online Mathematical Symbol Recognition using SVMs with Features from Functional Approximation", Electronic Proc. Mathematical User-Interfaces Workshop 2008, (MathUI 2008) http://www.activemath.org/workshops/MathUI/08/proceedings.

[5] O. Golubitsky and S. M. Watt, "Improved Classification through Runoff Elections", pp. 59-64, Proc. International Workshop on Document Analysis Systems, (DAS 2010), ACM Press.

[6] O. Golubitsky and S. M. Watt, "Online Stroke Modeling for Handwriting Recognition”, pp. 72-80, Proc. 18th International Conf. on Computer Science and Software Engineering, (CASCON 2008), IBM Canada.

[7] O. Golubitsky and S. M. Watt, "Online Computation of Similarity between Handwritten Characters", pp. C1-C10, Proc. Document Recognition and Retrieval XVI, (DRR XVI 2009), SPIE and IS\&T.

[8] O. Golubitsky, V. Mazalov and S. M. Watt, "Orientation-Independent Recognition of Handwritten Characters with Integral Invariants", pp. 252-261, Proc. Joint Conf. of ASCM 2009 and MACIS 2009: Asian Symposium of Computer Mathematics and Mathematical Aspects of Computer and Information Sciences, (ASCM 2009), COE Lecture Note Vol. 22, Kyushu University, ISSN 1881-4042.

[9] O. Golubitsky, V. Mazalov and S. M. Watt, "Towards Affine Recognition of Handwritten Mathematical Characters", pp. 35-42, Proc. Int'l Workshop on Document Analysis Systems, (DAS 2010), ACM Press.

[10] O. Golubitsky and S. M. Watt, "Confidence Measures in Recognizing Handwritten Mathematical Symbols", pp. 460-466, Proc. Conf. on Intelligent Computer Mathematics 2009: 16th Symposium on the Integration of Symbolic Computation and Mechanized Reasoning and 8th International Conf. on Mathematical Knowledge Management, (MKM 2009), Springer Verlag LNAI 5625.

[11] C. M. So and S. M. Watt, "Determining Empirical Properties of Mathematical Expression Use”, pp. 361-375, Proc. Fourth International Conf. on Mathematical Knowledge Management, (MKM 2005), Springer Verlag LNCS 3863.

[12] S. M. Watt, "An Empirical Measure on the Set of Symbols Occurring in Engineering Mathematics Texts", pp. 557-564, Proc. 8th IAPR International Workshop on Document Analysis Systems, (DAS 2008), IEEE Computer Society.

[13] E. Smirnova and S. M. Watt, "Context-Sensitive Mathematical Character Recognition", pp. 604-610, Proc. IAPR International Conf. on Frontiers in Handwriting Recognition, (ICFHR 2008), Cenparmi, Concordia University, ISBN 1-895193-03-6.

[14] S. M. Watt, "Mathematical Document Classification via Symbol Frequency Analysis", pp. 29-40, Proc. Towards Digital Mathematics Library, (DML 2008), Masaryk University, ISBN 978-80-210-4658-0.

[15] V. Mazalov and S. Watt, "Digital Ink Compression via Functional Approximation", Proc. 12th International Conf. on Frontiers in Handwriting Recognition, (ICFHR 2010), to appear. 